\title{
The effect of magnetic and non-magnetic ion damage on the surface state in $\mathrm{SmB}_{6}$
}

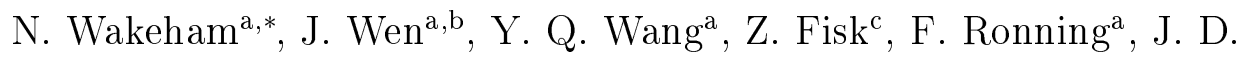 \\ Thompson $^{\mathrm{a}}$ \\ ${ }^{a}$ Los Alamos National Laboratory \\ ${ }^{b}$ Lanzhou University, Gansu, China \\ ${ }^{c}$ Dept. of Physics and Astronomy, University of California, Irvine
}

\begin{abstract}
$\mathrm{SmB}_{6}$ is a Kondo insulator with a band structure that is topologically distinct from the vacuum. This is theoretically predicted to produce metallic topological surface states that are robust to perturbations that do not break time reversal symmetry, such as non-magnetic defects. However, the surface state may be destroyed by an impurity with a sufficiently large magnetic moment. In order to test this prediction we show measurements of the resistance of the surface state of single crystals of $\mathrm{SmB}_{6}$ with varying levels of damage induced by magnetic and non-magnetic ion irradiation. We find that at a sufficiently high concentration of damage the surface state reconstructs below an amorphous damaged layer, whether the damage was caused by a magnetic or non-magnetic ion.
\end{abstract}

Keywords: , $\mathrm{SmB}_{6}$, Topological Kondo Insulator, Magnetic, Damage

\section{Introduction}

$\mathrm{SmB}_{6}$ is a known Kondo insulator in which hybridization of the $f$ and conduction electrons opens a small energy gap at the Fermi energy Kasuya (1994). This produces a diverging resistivity as temperature decreases, as expected, but the resistivity saturates at around $3 \mathrm{~K}$. The band structure of $\mathrm{SmB}_{6}$ was recently predicted to be topologically non-trivial Dzero et al.

*Email: nwakeham@lanl.gov

Preprint submitted to Journal of Magnetism and Magnetic Materials

July 2, 2015

(C) 2015. This manuscript version is made available under the Elsevier user license http://www.elsevier.com/open-access/userlicense/1.0/ 
(2010). At the interface between a topologically non-trivial insulator and the trivial vacuum the energy gap is predicted to close, giving rise to metallic surfaces states on the crystal Ando (2013); Hasan and Kane (2010); Qi and Zhang (2011). The low temperature resistivity saturation in $\mathrm{SmB}_{6}$ was recently shown to be because of metallic states on the surface Syers et al. (2014); Wolgast et al. (2013); Kim et al. (2013), but the topological nature of those states is still a matter of intense debate.

One of the predicted properties of a topologically protected surface state is that it is robust to the back-scattering of electrons by perturbations that do not break time reversal symmetry, such as non magnetic impurities and defects. However, magnetic impurities are predicted to cause a large reduction in the conductivity of the surface state. Indeed, recent work showed that bulk doping of $\mathrm{SmB}_{6}$ with magnetic Gd ions destroyed the surface state, while doping with non-magnetic Y ions did not Kim et al. (2014). However, this kind of bulk doping inevitably disrupts the bulk band structure of the material, as well as the surface. A method of perturbing the surface of the crystal while not affecting the bulk, is therefore desirable. In previous work on $\mathrm{SmB}_{6}$ the surface was damaged while the bulk was left pristine using irradiation with $\mathrm{Xe}$ and Ar ions. The robustness of the surface state to this non-magnetic perturbation was tested by measuring the resistance of the sample after damage to progressively deeper into the crystal Wakeham et al. (2015). The crystals were damaged to a final depth of greater than $200 \mathrm{~nm}$ and therefore significantly deeper than current estimates of the intrinsic surface state which is of order $10 \mathrm{~nm}$ Roy et al. (2014); Alexandrov et al. (2013, 2015); Neupane et al. (2013); Jiang et al. (2013). In that work the data were consistent with a model in which the surface state reconstructed below a poorly conducting damaged layer. This is consistent with the theoretical predictions for a topological insulator Zhao et al. (2010); Roy et al. (2014).

Here, we report on further work to measure the resistance of $\mathrm{SmB}_{6}$ single crystals damaged through ion irradiation. In contrast to simple expectations irradiation with magnetic Fe ions does not destroy the surface state, and we present evidence that the surface state is still reconstructed below the damaged layer. In addition, through low concentration damage of the surface state with non-magnetic ions, we show that the low temperature resistance of the sample initially increases and then at higher concentrations decreases as before. 


\section{Method}

Single crystals of $\mathrm{SmB}_{6}$ with approximate dimensions of $400 \mu \mathrm{m}$ x $150 \mu \mathrm{m}$ were grown using Al flux and polished down to around $100 \mu \mathrm{m}$ thick. In order to measure the resistance $R$ of the samples, four Pt wires were spot welded to the top face of the crystal. A four point resistance measurement was then performed using an AC resistance bridge. The surface damage of the samples with magnetic ions was produced using $\mathrm{Fe}^{+}$, and non-magnetic damage with $\mathrm{Ar}^{+}$ions. The depth and level of damage was calculated using the SRIM Monte Carlo code in the full cascade mode Ziegler et al. (2010). The ion, acceleration energy, fluence and exposure time for each ion exposure is show in table 1 . The stated depth of damage was defined as the depth at which the damage level is half of the maximum damage, as discussed previously Wakeham et al. (2015). The resistance of each sample was measured as a

\begin{tabular}{cccccc}
\hline \hline $\begin{array}{c}\text { Depth } \\
(\mathrm{nm})\end{array}$ & $\begin{array}{c}\text { Damage } \\
(\mathrm{DPA})\end{array}$ & Ion & $\begin{array}{c}\text { Energy } \\
(\mathrm{keV})\end{array}$ & $\begin{array}{c}\text { Ion fluence } \\
\left(\mathrm{cm}^{-2}\right)\end{array}$ & $\begin{array}{c}\text { Time } \\
(\mathrm{s})\end{array}$ \\
\hline \hline 17.5 & 1 & $\mathrm{Fe}^{+}$ & 20 & $7 \times 10^{14}$ & 45 \\
105 & 1 & $\mathrm{Fe}^{+}$ & 160 & $7 \times 10^{14}$ & 62 \\
\hline 160 & 0.001 & $\mathrm{Ar}^{+}$ & 200 & $1.1 \times 10^{12}$ & 10 \\
160 & 0.01 & $\mathrm{Ar}^{+}$ & 200 & $1.1 \times 10^{13}$ & 112 \\
160 & 0.1 & $\mathrm{Ar}^{+}$ & 200 & $1.1 \times 10^{14}$ & 1129 \\
160 & 1 & $\mathrm{Ar}^{+}$ & 200 & $1.1 \times 10^{15}$ & 7200 \\
\hline
\end{tabular}

Table 1: Parameters used in the ion-irradiation of $\mathrm{SmB}_{6}$. The damage is quantified in units of displacements per atom (DPA).

function of temperature down to $1.8 \mathrm{~K}$, after the crystals were damaged on the top surface, and then again after damage to the bottom surface. This was repeated for each round of damage, with the leads permanently attached for the duration of the experiment. Three samples were damaged with magnetic ions and another sample was damaged with non magnetic ions. 


\section{Results}

Figure 1 shows the sheet resistance, $R_{s}=R 2 w / l$, where $w$ is the width of the sample and $l$ is the distance between voltage contacts, as a function of temperature $T$ for one of the samples damaged by irradiation with $\mathrm{Fe}^{+}$ions to progressively greater depths into the surface, as described above. Note

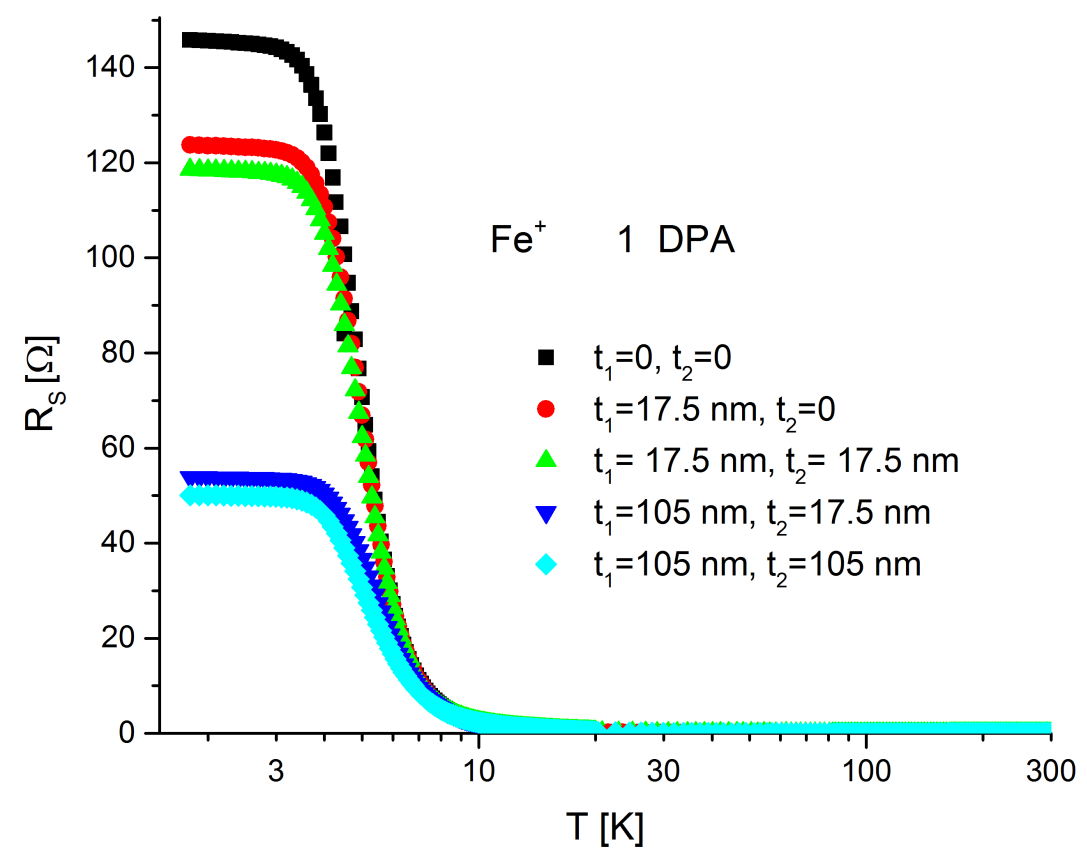

Figure 1: Temperature dependence of the sheet resistance $R_{s}$ of one of the $\mathrm{SmB}_{6}$ crystals after differing depths of $\mathrm{Fe}^{+}$ion-irradiation damage to each face. $t_{1}$ is the depth of damage to the top face of the sample (to which contacts were made), $t_{2}$ is the depth of damage to the bottom face.

that after the ion damage the low temperature saturation in the resistance is still present in the damaged sample, but the magnitude is monotonically reduced. Significant damage to $\mathrm{SmB}_{6}$ is known to destroy the Kondo gap, making the damaged region a poor conductor Karkin et al. (2007). This means that one effect of damaging the surface of $\mathrm{SmB}_{6}$ is to produce a new conducting channel in the sample. The important question though is the fate of the intrinsic surface state after the damage. To investigate this it is useful to take the inverse of the residual sheet resistance at low temperatures to give the residual sheet conductance $G_{S 0}$. For all three samples this is plotted 
in Fig. 2 as a function of $d$, the sum of the depth of damage to the top and bottom surfaces. Data from previous work on the effect of non-magnetic ion damage with Xe and Ar ions are also shown in Fig. 2 for comparison.

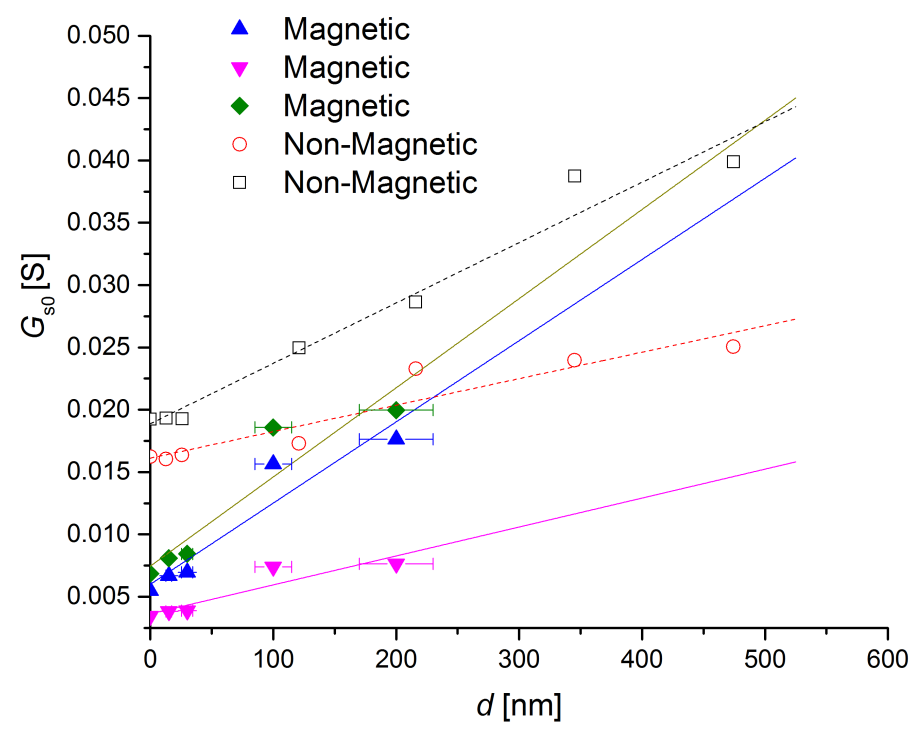

Figure 2: Residual sheet conductance $G_{S 0}$ of $\mathrm{SmB}_{6}$ as a function of the effective depth of ion-radiation damage $d$, given by the sum of the damage depth to the top and bottom faces. Closed symbols are data for damage with magnetic $\mathrm{Fe}^{+}$ions on 3 different samples, and open symbols are previously published data of damage with non-magnetic Ar and Xe ions on 2 different samples Wakeham et al. (2015). Lines are fits to the formula $G_{S 0}=1 / R_{S S}+d / 2 \rho_{D L}$, where $\rho_{D L}$ is the resistivity of the damaged layer.

A scenario in which the surface state is not destroyed, but reconstructs below the damaged layer, can be modelled using the equation $G_{S 0}=1 / R_{S S}+$ $d / 2 \rho_{D L}$. This describes a parallel resistor model in which the first term represents the intrinsic surface state with sheet resistance $R_{S S}$, given by the sheet resistance of the undamaged sample at low temperature. This term is assumed to be independent of $d$. The second term represents the poorly conducting damaged region and is proportional to the damage depth. This equation has been fitted to the experimental data in Fig 2, where $\rho_{D L}$ is the resistivity of the damaged layer and is a fitted parameter calculated to be in the range $0.7-2.2 \mathrm{~m} \Omega \mathrm{cm}$, which is in reasonable agreement with previous ion and neutron damage studies Wakeham et al. (2015); Karkin et al. (2007). 
While there are deviations in the data from the fitted model, importantly the data are more consistent with this equation than a fit that is linear in $d$ with a zero intercept. A zero intercept would be the expected behaviour had the surface state been destroyed by the damage. Therefore, we conclude that the surface state has not been destroyed by the magnetic ion damage, but instead the state reconstructs below the damaged layer, as seen in our work on non-magnetic damage. Studies to greater depths may be useful, though the data shown support our interpretation. Deviations of the experimental data from our fitted model may arise from inhomogeneity in the damage across the surface, or the effects of a damage profile as a function of the depth that is more complicated than the simple step function assumed here. Work to further investigate these inhomogeneities is ongoing. The variation in the sheet resistance between samples is consistent with values reported in the literature. This may be the result of intrinsic variations in sample quality or a sensitivity of the sample surface to mechanical treatment. These two factors are likely to also account for the variation in the fitted resistivity of the damaged region.

The insensitivity of the surface state in $\mathrm{SmB}_{6}$ to the magnetic damage produced by the Fe ion irradiation is perhaps surprising given the expectation that a topological surface state would be destroyed by time reversal symmetry breaking perturbations. However, this result is consistent with measurements of the resistivity under an applied magnetic field. It has been shown that the application of large magnetic fields up to $60 \mathrm{~T}$ is insufficient to close the Kondo gap, or destroy the low temperature saturation of resistance Cooley et al. (1999). This implies that the direction of the spin of the surface state electrons may be insensitive to magnetic field and therefore to magnetic impurities. Therefore, the results presented here are still consistent with a topological surface state in $\mathrm{SmB}_{6}$. It must also be considered that perhaps the surface state reconstructs to a region in the sample that is free of magnetic impurities. This scenario will have to be investigated in future work.

Our work implies that the physical interface between the $\mathrm{SmB}_{6}$ and the vacuum may not be the location of the conductive surface state, depending on the treatment of the surface. This may be relevant to surface sensitive measurements such as the photoemission or scanning tunneling spectroscopy. In addition the location of the conducting state may be important in constructing heterostructures of $\mathrm{SmB}_{6}$ with magnetic or superconducting layers as has been proposed to search for exotic states such as Majorana fermions, 
for example Hou et al. (2012); Affleck and Giuliano (2013); Williams et al. (2012).

In the work discussed so far the damage caused has been sufficiently concentrated to destroy the Kondo gap in the damaged layer and lead to a reconstruction of the surface state. It is now interesting to consider the effect of damage levels at lower concentrations, and whether there is any increase in scattering as a result of the damage. So finally, figure 3 shows the sheet resistance as a function of temperature of an $\mathrm{SmB}_{6}$ crystal damaged with $\mathrm{Ar}^{+}$ ions to $160 \mathrm{~nm}$ with a progressively greater concentration of damage. Note

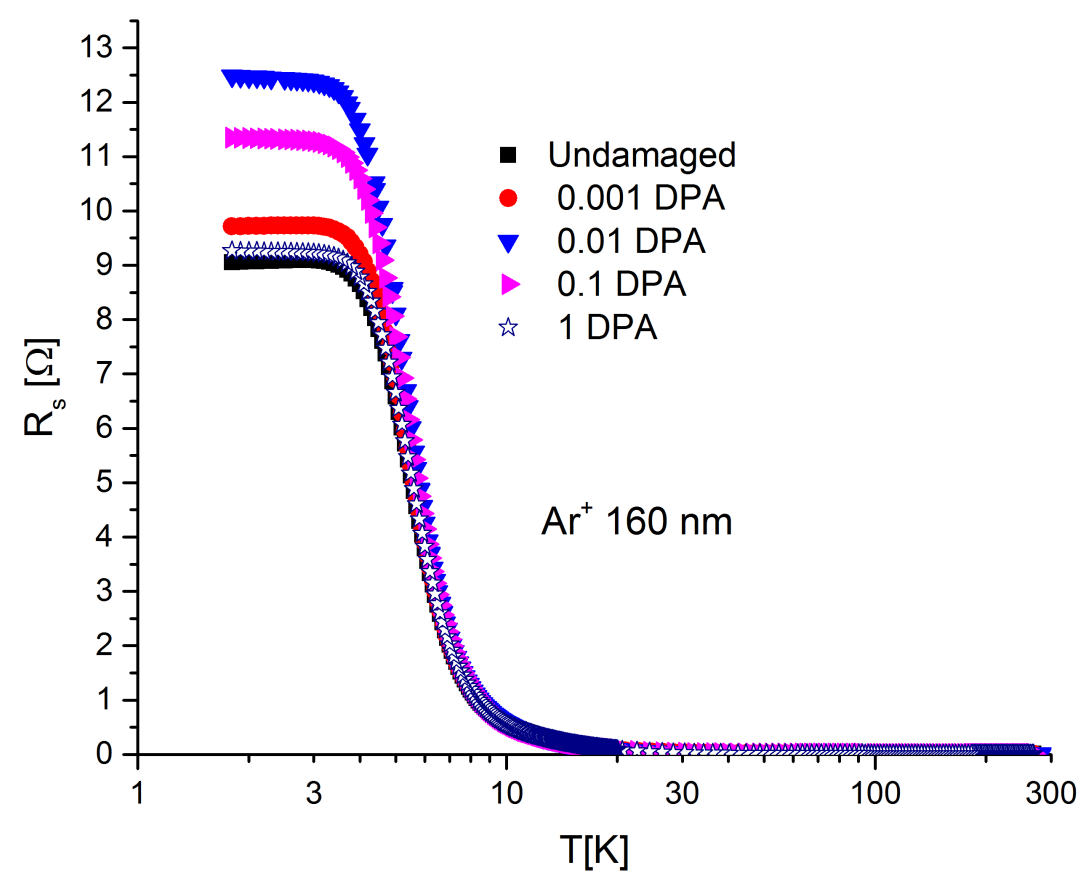

Figure 3: Temperature dependence of the sheet resistance $R_{s}$ of an $\mathrm{SmB}_{6}$ crystal after differing concentrations of $\mathrm{Ar}^{+}$ion-irradiation damage to a depth of $160 \mathrm{~nm}$.

again that the low temperature resistance saturation is not destroyed but now the magnitude is no longer a monotonic function of the damage. At low levels of damage the sheet resistance is modestly increased by the damage, likely because of an increase in small angle scattering of the surface state electrons. Once the damage becomes sufficiently concentrated the resistance begins to fall because of the effect of the addition of the conduction channel from the heavily damaged layer, as discussed above. These data suggest that 
a low concentration of non-magnetic disorder may have a small effect on the conductivity of the intrinsic surface state, but once the damage is sufficiently large the surface state reconstructs below the damage.

\section{Conclusion}

In conclusion we have shown that heavy irradiation of the surface of $\mathrm{SmB}_{6}$ crystals with $\mathrm{Fe}^{+}$ions does not cause the destruction of the intrinsic surface state, but instead, as in the case of non-magnetic ion damage, the surface state reconstructs below the poorly conducting damaged layer. While this implies some robustness of the surface state to magnetic damage, this is qualitatively consistent with measurements under an applied magnetic field. This is not inconsistent with the surface state being topological in nature, it merely implies that the spin direction of the surface electrons is relatively insensitive to magnetic field. Light damage by non-magnetic ions was shown to cause an increase in the sheet resistance of the crystal, likely because of increased scattering, but at heavier irradiation doses the resistance was seen to decrease as before. This implies some sensitivity of the surface state to disorder which will require further investigation. These results have important implications for future studies of $\mathrm{SmB}_{6}$, and may also be relevant to technological uses of $\mathrm{SmB}_{6}$ in heterostructures with magnetic layers, for example.

\section{Acknowledgements}

NW acknowledges the support of the Los Alamos National Laboratory LDRD program. The work of FR and JT was performed under the auspices

of the U.S. Department of Energy, Office of Science. The support for JW was partially provided by the China Scholarship Council. The Ion implantation facility was partially supported by the Center for Integrated Nanotechnologies (CINT), a DOE nanoscience user facility jointly operated by Los Alamos and Sandia National Laboratories.

Affleck, I., Giuliano, D., Jun. 2013. Topological superconductor-Luttinger liquid junctions. J. Stat. Mech. Theory Exp. 2013 (06), P06011.

Alexandrov, V., Coleman, P., Erten, O., Apr. 2015. Kondo Breakdown in Topological Kondo Insulators. Phys. Rev. Lett. 114 (17), 177202. 
Alexandrov, V., Dzero, M., Coleman, P., Nov. 2013. Cubic Topological Kondo Insulators. Phys. Rev. Lett. 111 (22), 226403.

Ando, Y., Oct. 2013. Topological Insulator Materials. J. Phys. Soc. Japan 82 (10), 102001.

Cooley, J.C. and Mielke, C.H. and Hults, W.L. and Goettee, J.D. and Honold, M.M. and Modler, R.M. and Lacerda, A. and Rickel, D.G. and Smith, J.L. Feb. 1999. High Field Gap Closure in the Kondo Insulator $\mathrm{SmB}_{6}$. Journ. Supercond. 12 (1) 171.

Dzero, M., Sun, K., Galitski, V., Coleman, P., Mar. 2010. Topological Kondo Insulators. Phys. Rev. Lett. 104 (10), 106408.

Hasan, M. Z., Kane, C. L., Nov. 2010. Colloquium: Topological insulators. Rev. Mod. Phys. 82 (4), 3045-3067.

Hou, C.-Y., Shtengel, K., Refael, G., Goldbart, P. M., Oct. 2012. Ettingshausen effect due to Majorana modes. New J. Phys. 14 (10), 105005.

Jiang, J., Li, S., Zhang, T., Sun, Z., Chen, F., Ye, Z. R., Xu, M., Ge, Q. Q., Tan, S. Y., Niu, X. H., Xia, M., Xie, B. P., Li, Y. F., Chen, X. H., Wen, H. H., Feng, D. L., Dec. 2013. Observation of possible topological in-gap surface states in the Kondo insulator $\mathrm{SmB}_{6}$ by photoemission. Nat. Commun. 4, 3010.

Karkin, A., Akshentsev, Y., Goshchitskii, B., Sep. 2007. Insulator-to-metal transition in $\mathrm{SmB}_{6}$ induced by neutron irradiation. Phys. C Supercond. 460-462, 811-812.

Kasuya, T., 1994. Gap state in $\mathrm{YbB}_{12}$ and $\mathrm{SmB}_{6}$ : real Kondo insulators. Europhys. Lett. 26 (277), 2-7.

Kim, D. J., Thomas, S., Grant, T., Botimer, J., Fisk, Z., Xia, J., Nov. 2013. Surface hall effect and nonlocal transport in $\mathrm{SmB}_{6}$ : evidence for surface conduction. Sci. Rep. 3, 3150.

Kim, D. J., Xia, J., Fisk, Z., March 2014. Topological surface state in the Kondo insulator samarium hexaboride. Nat. Mater. 13, 466-70. 
Neupane, M., Alidoust, N., Xu, S.-Y., Kondo, T., Ishida, Y., Kim, D. J., Liu, C., Belopolski, I., Jo, Y. J., Chang, T.-R., Jeng, H.-T., Durakiewicz, T., Balicas, L., Lin, H., Bansil, A., Shin, S., Fisk, Z., Hasan, M. Z., Dec. 2013. Surface electronic structure of the topological Kondo-insulator candidate correlated electron system $\mathrm{SmB}_{6}$. Nat. Commun. 4, 2991.

Qi, X.-L., Zhang, S.-C., Oct. 2011. Topological insulators and superconductors. Rev. Mod. Phys. 83 (4), 1057-1110.

Roy, B., Sau, J. D., Dzero, M., Galitski, V., Oct 2014. Surface Theory of a Family of Topological Kondo Insulators. Phys. Rev. B 90, 155314.

Syers, P., Kim, D., Fuhrer, M. S., Paglione, J., Mar. 2015. Tuning bulk and surface conduction in topological Kondo insulator $\mathrm{SmB}_{6}$. Phys. Rev. Lett. $114,056803$.

Wakeham, N., Wang, Y. Q., Fisk, Z., Ronning, F., Thompson, J. D., Feb. 2015. Surface state reconstruction in ion-damaged $\mathrm{SmB}_{6}$. Phys. Rev. B $91(8), 085107$.

Williams, J. R., Bestwick, A. J., Gallagher, P., Hong, S. S., Cui, Y., Bleich, A. S., Analytis, J. G., Fisher, I. R., Goldhaber-Gordon, D., Jul. 2012. Unconventional Josephson Effect in Hybrid Superconductor-Topological Insulator Devices. Phys. Rev. Lett. 109 (5), 056803.

Wolgast, S., Kurdak, C., Sun, K., Allen, J. W., Kim, D.-J., Fisk, Z., Nov. 2013. Low-temperature surface conduction in the Kondo insulator $\mathrm{SmB}_{6}$. Phys. Rev. B 88 (18), 180405.

Zhao, E., Zhang, C., Lababidi, M., Nov. 2010. Mott scattering at the interface between a metal and a topological insulator. Phys. Rev. B 82 (20), 205331.

Ziegler, J. F., Ziegler, M. D., Biersack, J. P., 2010. SRIM - The stopping and range of ions in matter (2010). Nucl. Instruments Methods Phys. Res. Sect. B Beam Interact. with Mater. Atoms 268, 1818-1823. 